Trauma Berufskrankh 2009 · 11 [Suppl 2]: 179-182

DOI 10.1007/s10039-008-1468-8

Online publiziert: 7. Februar 2009

(c) Springer Medizin Verlag 2009
J. Heineck · S. Rein

Klinik für Unfall- und Wiederherstellungschirurgie, Universitätsklinik, Dresden

\section{Periprothetische kniegelenknahe Fraktur} Klassifikation der operativen Therapie

\section{Ausgangssituation}

Die Inzidenz periprothetischer Frakturen bei liegender Knietotalendoprothese (Knie-TEP) ist insgesamt niedrig. Sie nimmt vom Femur über die Tibia zur Patella ab $[1,3]$. Das Patientenalter ist überdurchschnittlich hoch. Eine schwere Allgemeinerkrankung (ASA 3) weisen 35\% der Patienten auf. Die Knochenqualität ist in der Regel schlecht.

Meist liegt ein Oberflächenersatz vor, oft sind aber auch intramedulläre Implantate (Prothesenstiel, gleichseitige HüftTEP) vorhanden. Die betroffenen Patienten sind meist nicht in der Lage, postoperativ eine Teilbelastung einzuhalten.

\section{Behandlungsziele}

Um die Prothese möglichst dauerhaft zu erhalten, sollte eine Ausheilung der Fraktur in anatomischer Stellung erreicht werden. Zum Erhalt der Prothesenbeweglichkeit ist zumindest eine Übungsstabilität der Osteosynthese zu fordern. Aufgrund des Allgemeinzustandes der Patienten sollte das Operationstrauma möglichst gering sein.

Diese zur schlechten Ausgangssituation in Widerspruch stehenden ehrgeizigen Behandlungsziele resultieren letztlich in einer hohen Komplikationsrate von 25-27\% in der Literatur [3].

\section{Implantate}

Der Markt für Endoprothesen hält modulare Revisionsimplantate bis hin zum totalen Femurersatz bereit ( $\bullet$ Abb. 1). An
Osteosynthesematerial kommen bevorzugt winkelstabile Platten und ggf. Marknägel zum Einsatz. Auch auf Cerclagen kann häufig nicht verzichtet werden. Diese können bei modernen Plattensystemen an die Platte gekoppelt werden, sodass sie auf den schrägen Oberflächen der Metaphyse nicht abrutschen und sich so lockern. Aufgrund der häufig vorliegenden knöchernen Defekte kann der Einsatz von sowohl Knochenzement als auch autologem oder allogenem Knochen als auch Knochenersatzmaterialien erforderlich werden.

\section{Patellafrakturen}

Sie können nach Ortiguera u. Berry [4] eingeteilt werden (• Tab. 1). Diese fanden in einem Kollektiv von $12.464 \mathrm{~Pa}$ tienten 78 periprothetische Patellafrakturen.

Das Primat der Therapie ist die Rekonstruktion des Streckapparats, das $\mathrm{Pa}$ tellaimplantat zu erhalten, ist sekundär. Typ 2 wird somit in der Regel operiert. Bei der Osteosynthese kommen konventionelle Zuggurtungen und Schraubenosteosynthesen zum Einsatz (• Abb. 2).

\begin{tabular}{|ll|}
\hline Tab. 1 & Einteilung der Patellafrakturen. (Nach [4]) \\
\hline $\begin{array}{l}\text { Typ } \\
1\end{array}$ & $\begin{array}{l}\text { Charakteristika } \\
\text { Streckapparat intakt } \\
\text { Implantat fest }\end{array}$ \\
\hline 2 & Streckapparat zerstört \\
\hline 3 & $\begin{array}{l}\text { Streckapparat intakt } \\
\text { Implantat locker }\end{array}$ \\
\hline 3A & Ausreichende Knochendicke \\
\hline 3B & Nicht ausreichende Knochendicke \\
\hline
\end{tabular}

\begin{tabular}{|c|c|c|c|}
\hline Frakturtyp & Anatomische Charakteristika & Subtyp & Prothesencharakteristika \\
\hline \multirow[t]{3}{*}{ I } & \multirow[t]{3}{*}{ Tibiaplateau } & A & Prothese fest \\
\hline & & B & Prothese locker \\
\hline & & C & Intraoperativ \\
\hline \multirow[t]{3}{*}{ II } & \multirow[t]{3}{*}{ Dem Schaft anliegend } & A & Prothese fest \\
\hline & & B & Prothese locker \\
\hline & & C & Intraoperativ \\
\hline \multirow[t]{3}{*}{ III } & \multirow[t]{3}{*}{ Fraktur distal der Prothese } & A & Prothese fest \\
\hline & & B & Prothese locker \\
\hline & & C & Intraoperativ \\
\hline \multirow[t]{3}{*}{ IV } & \multirow[t]{3}{*}{ Tibiatuberkel } & A & Prothese fest \\
\hline & & B & Prothese locker \\
\hline & & C & Intraoperativ \\
\hline
\end{tabular}




\section{Knieverletzungen}
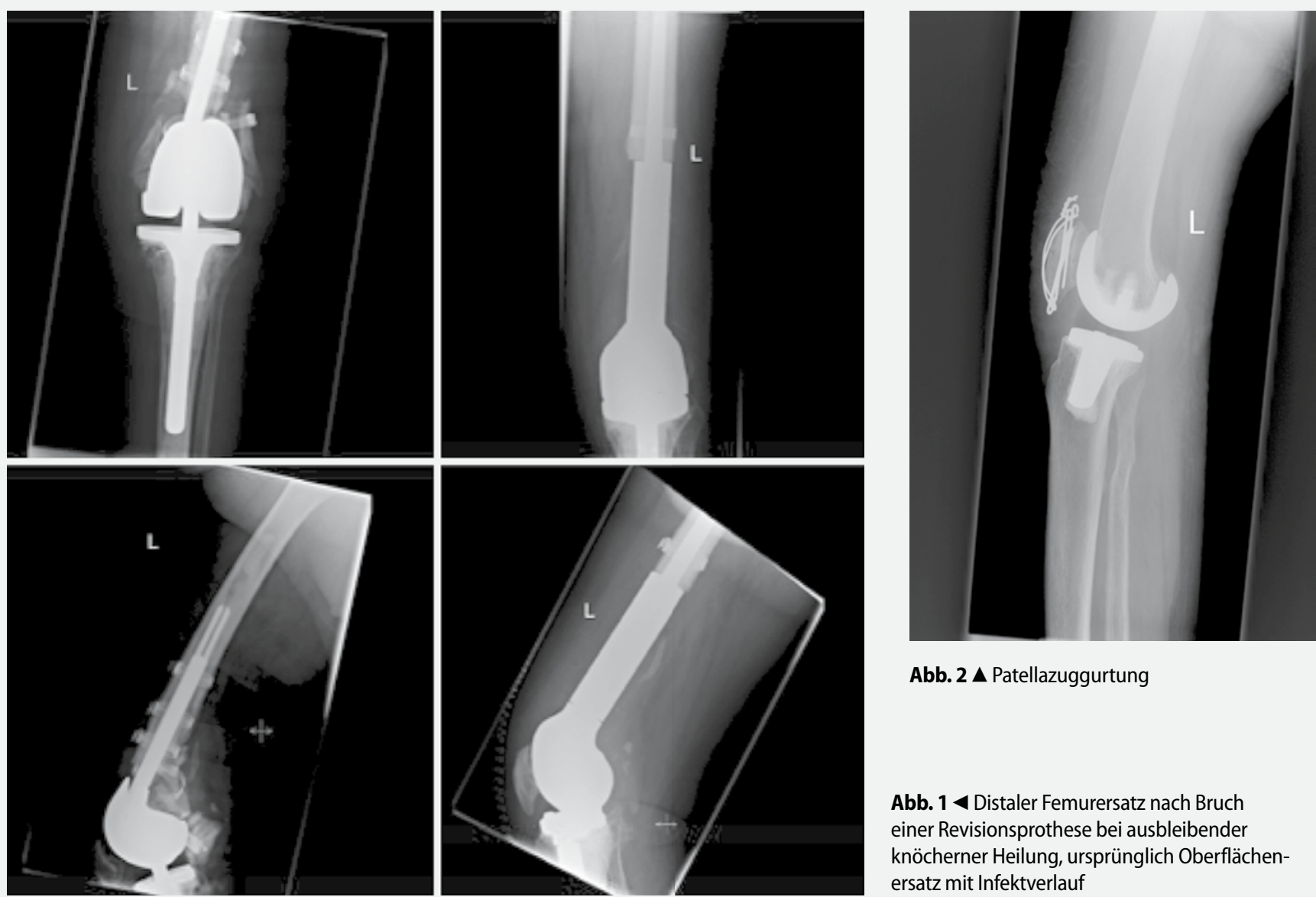

Abb. $2 \Delta$ Patellazuggurtung

Abb. 14 Distaler Femurersatz nach Bruch einer Revisionsprothese bei ausbleibender knöcherner Heilung, ursprünglich Oberflächenersatz mit Infektverlauf

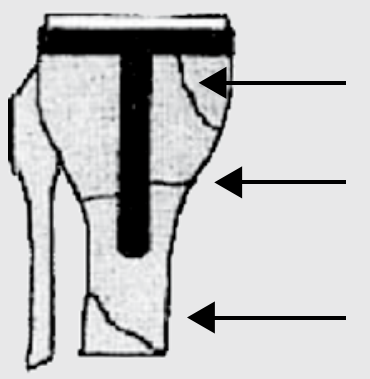

a.-p.

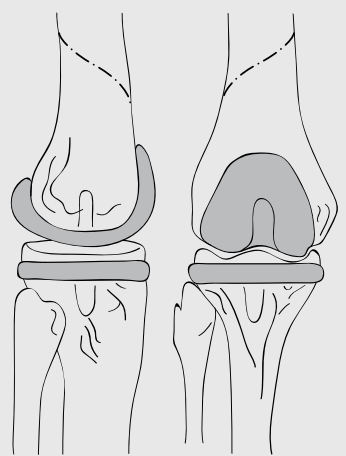

Typ I

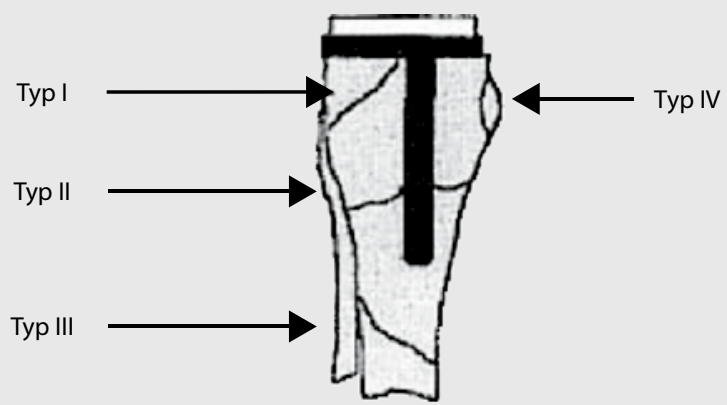

Lateral
Abb. 34 Klassifikation der Tibiafrakturen. (Nach [2]), s. a. Tab. 2

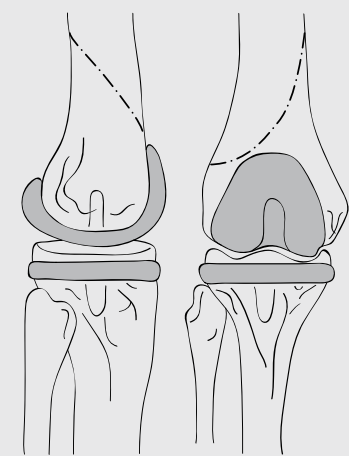

Typ II

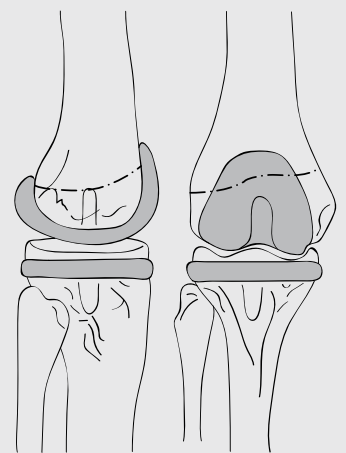

Typ III
Abb. $4 \varangle$ Su-Klassifikation der periprothetischen Femurfrakturen. (Nach [7]) 
Tab. 3 Klassifikation der periprothe-

tischen Femurfrakturen nach Rorabeck

u. Taylor. (Nach [6])

Typ Charakteristika

1 Implantat fest Implantat undisloziert

2 Implantat fest Implantat $>5 \mathrm{~mm}$ disloziert

3 Implantat gelockert Implantat disloziert oder undisloziert

Die Komplikationsrate bei operativem Vorgehen ist mit bis zu 42\% Reoperationen [4] allerdings sehr hoch. Deshalb sollte bei intaktem Streckapparat und festem Implantat (Typ 1) eine konservative Therapie durchgeführt werden. Hier wurden nur selten Therapieversager beobachtet. Bei intaktem Streckapparat und gelockertem (Typ 3) Implantat hängt die Therapie von den Beschwerden des Patienten $a b$, beschwerdefreie Patienten sollten ebenfalls konservativ behandelt werden. Abrissfrakturen der Tuberositas tibiae sind nur bei größeren knöchernen Fragmenten gut zu refixieren. Bei kleinen Fragmenten oder Avulsionen der Patellarsehne sind oft Sehnenautografts oder Allografts erforderlich.

\section{Tibia und Femur}

Die Tibiafrakturen werden nach $\mathrm{Fe}$ lix et al. [2] ( $n=102$ Patienten) eingeteilt (• Abb. 3).

Bei fest sitzendem Implantat erfolgt in der Regel eine Osteosynthese. Die Frakturen vom Typ 1, bei denen es sich meist um Ermüdungsbrüche des medialen Tibiakopfs handelt, können in Ausnahmefällen konservativ behandelt werden. Hierbei ist allerdings mit Bewegungsdefiziten zu rechnen. Bei gelockertem Implantat wird ein Prothesenwechsel durchgeführt. In Ausnahmefällen, meist bei jüngeren Patienten, können zunächst die Fraktur zur Ausheilung gebracht und dann das Implantat gewechselt werden. Der Sinn dieses Vorgehens liegt in der Vermeidung von übergroßen Revisionsimplantaten.

Aktuelle Klassifikationen der periprothetischen Femurfrakturen sind die Einteilungen nach Rorabeck u. Taylor [6] sowie Su et al. [7] ( $\bullet$ Abb.4). Rorabeck u. Taylor [6] unterscheiden 3 Typen (• Tab. 3).

Trauma Berufskrankh 2009 • 11 [Suppl 2]: 179-182 DOI 10.1007/s10039-008-1468-8

(c) Springer Medizin Verlag 2009

\section{J. Heineck · S. Rein \\ Periprothetische kniegelenknahe Fraktur. Klassifikation der operativen Therapie}

\section{Zusammenfassung}

Periprothetische Frakturen treten bei Patienten mit einer Knietotalendoprothese selten auf. Die Betroffenen sind meist sehr alt, leiden häufig an einer schweren Allgemeinerkrankung, und ihre Knochenqualität ist in der Regel schlecht. Zudem sind sie meist nicht in der Lage, postoperativ eine Teilbelastung einzuhalten. Die kaum zu erreichenden Ziele der Behandlung sind eine Ausheilung der Fraktur in anatomischer Stellung, Übungsstabilität der Osteosynthese und ein möglichst geringes Operationstrauma. Die Komplikationsrate ist aufgrund der schlechten Ausgangsbedingungen hoch (25-27\%). Patellafrakturen Typ 1 sollten konservativ, Typ-2-Brü-

che operativ behandelt werden. Bei Typ-3Frakturen hängt das Vorgehen von den Beschwerden des Patienten ab. Bei Tibia-/Femurfrakturen mit fest sitzendem Implantat sollte eine Osteosynthese durchgeführt werden, bei gelockertem Implantat ist ein Prothesenwechsel indiziert. Typ-1-Frakturen können in Ausnahmefällen konservativ behandelt werden.

\section{Schlüsselwörter}

Periprothetische Fraktur $\cdot$ Knietotalendoprothese $\cdot$ Ausheilung in anatomischer Stellung • Übungsstabilität der Osteosynthese . Komplikationsrate

\section{Periprosthetic knee fracture. Classification of surgical therapy}

\section{Abstract}

Periprosthetic fractures rarely occur in patients with total knee endoprosthesis, and when they do, the patients are usually of advanced age and suffering from severe systemic disease with poor bone quality. Moreover, they are generally unable to partially bear weight postoperatively. The almost unattainable goals of therapy are healing of the fracture in an anatomic position, mobilisation of the osteosynthesis and as little surgical trauma as possible. The complication rate is high $(25-27 \%)$ due to the poor initial conditions. Type 1 patella fractures should be treated conservatively, while type 2 frac- tures should be treated surgically. In the case of type 3 fractures, the therapeutic approach depends on the patient's symptoms. Osteosynthesis is the method of choice in tibia/femur fractures with fixed implants, while prosthesis replacement is indicated in lose implants. In exceptional cases, type 1 fractures can be treated conservatively.

\section{Keywords}

Periprosthetic fracture - Total knee endoprosthesis - Healing in anatomic position · Mobilisation . Complication rate 


\section{Knieverletzungen}

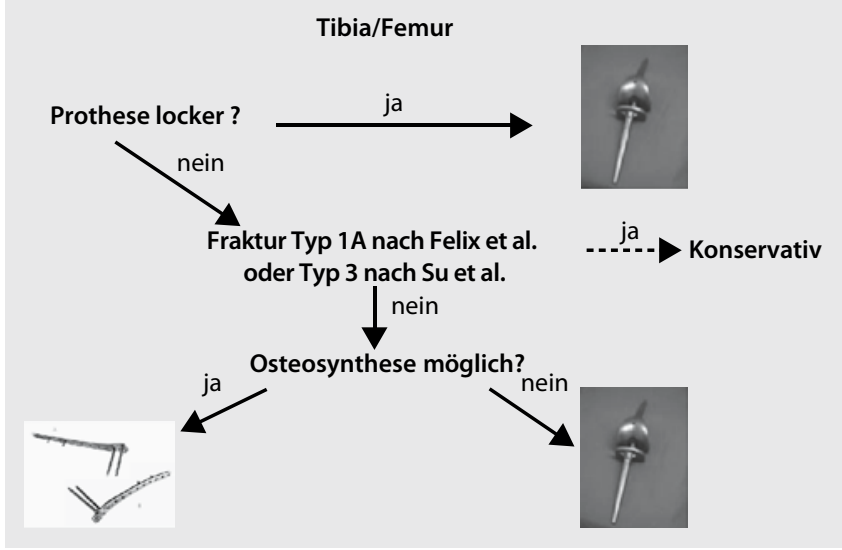

Abb. $5<$ Algorithmus der Behandlung bei periprothetischen Femur- und Tibiafrakturen

Das oft als Risikofaktor eingeschätzte anteriore femorale Notching zeigte sich in einer größeren klinischen Serie nicht als solcher [5]. Als Grund hierfür ist ein knöchernes Remodelling zu sehen. Den therapeutischen Algorithmus zeigt $\bullet$ Abb. 5. In Ausnahmefällen, insbesondere bei jüngeren Patienten kann auch hier zunächst eine Ausheilung der Fraktur angestrebt werden, um übergroße Revisionsimplantate $\mathrm{zu}$ vermeiden.

\section{Fazit für die Praxis}

Bei in großer Zahl und Variation vorhandenen Implantaten ist die Problematik der periprothetischen Femurfraktur hauptsächlich im schlechten Allgemeinzustand der Patienten sowie der schlechten Knochenheilung und den oft vorhandenen knöchernen Defekten zu sehen. Da dieses Problem nach wie vor ungelöst ist, ist mit einer wesentlichen Reduktion der hohen Komplikationsrate in nächster Zeit nicht zu rechnen. Insgesamt ist die Inzidenz der periprothetischen Femurfrakturen glücklicherweise jedoch niedrig.

\section{Korrespondenzadresse}

\section{Dr. J. Heineck}

Klinik für Unfall- und Wiederherstellungschirurgie, Universitätsklinik, Fetscherstraße 74, 01307 Dresden

Jan.Heineck@uniklinikum-dresden.de

Interessenkonflikt. Der korrespondierende Autor gibt an, dass kein Interessenkonflikt besteht.

\section{Literatur}

1. Diehl P, Burgkart R, Klier T et al (2006) Periprothetische Frakturen nach Knietotalendoprothetik. Orthopäde 35:961-974

2. Felix NA, Stuart MJ, Hanssen AD (1997) Periprosthetic fractures of the tibia associated with total knee arthroplasty. Clin Orthop 345:113-124

3. Mittlmeier T, Stöckle U, Perka C, Schaser KD (2005) Periprothetische Frakturen nach Knietotalendoprothetik. Unfallchirurg 108:481-496

4. Ortiguera CJ, Berry DJ (2004) Patellar fracture after total knee arthroplasty. J Bone Joint Surg Am 84A:532-540

5. Ritter MA, Faris PM, Keating EM (1988) Anterior femoral notching and ipsilateral supracondylar femur fracture in total knee arthroplasty. Orthop Clin North Am 30(2):209-214

6. Rorabeck CH, Taylor JW (1999) Classification of periprosthetic fracture complicating total knee arthroplasty. Orthop Clin North Am 30:209-214

7. Su ET, DeWal H, Di Cesare PE (2004) Periprosthetic femoral fractures above knee replacements. J Am Acad Orthop Surg 12:12-20 


\section{Murnauer Unfalltagung, Murnau, 12. Juli 2008}

Veranstaltet vom Landesverband Südost der Deutschen Gesetzlichen Unfallversicherung (DGUV), München

Wissenschaftliche Leitung: Prof.Dr. Volker Bühren, Murnau

\section{Posttraumatische Osteitis - Diagnostik}

Posttraumatic osteomyelitis - Diagnosis

\section{Laboruntersuchungen zur Osteitisdiagnostik}

Laboratory tests to diagnose osteomyelitis

F. Wagner

187 Mikrobiologie bei Osteitis.

Evidenzbasierte Medizin

Microbiology in osteomyelitis. Evidence-based medicine G. Walter $\cdot$ W. Hirschberger $\cdot$ R. Hoffmann

193 Bildgebende Diagnostik der Osteitis.

Szintigraphie, PET oder MRT?

Imaging of posttraumatic osteomyelitis.

Scintigraphy, PET or MRI?

R. Linke $\cdot H$. Weidemann $\cdot$ M. Militz

\section{Behandlungsalgorithmen}

\section{Treatment algorithms}

203 Akute posttraumatische Osteitis. Ist die Osteosynthese zu retten?

Acute posttraumatic osteomyelitis.

Is it possible to save the osteosynthesis?

U.-J. Gerlach · C. Grimme · R. Schoop

207 Kontinuitätserhalt oder Segmentresektion. Entscheidungshilfen bei der Sanierung der Osteitis am Schaft langer Röhrenknochen

Preservation of bone continuity or segment resection. Decision-making support in long-bone diaphyseal osteitis A.H. Tiemann · R. Braunschweig · G.O. Hofmann
211 Weichteilmanagement und knöcherne Rekonstruktion

Soft tissue management and osseous reconstruction

V. Heppert · P. Herrmann · P. Thoele · M. Pelzer $\cdot$ C. Wagner

\section{Sozio-ökonomische "Resistenz"-} Entwicklung

Socio-economic resistance development

217 Osteitis. Vorgehen bei MRE-Infektion

Osteomyelitis. Management of MRSA infection

T. von Stein

222 Begutachtung der Osteitis

Expert opinion on posttraumatic osteomyelitis

M. Diefenbeck · R. Beickert · G.O. Hofmann

229 Kostenanalyse und DRG-Vergütung der Osteitiskomplexbehandlung

Cost analysis and DRG reimbursement

of complex osteitis treatment

A. Scholz · M. Glombitza

232 Posttraumatische Osteitis. Behandlungs-

konzepte für die rationale Antibiotikatherapie

Posttraumatic osteitis. Antibiotic stewardship

for effective antimicrobial therapy

F. Kipp

\section{Schlussbemerkungen Closing remarks}

\section{Zusammenfassung des Tagungsprogramms}

Conference program summary

H.G.K. Schmidt

\section{Verschiedenes Miscellaneous}

\section{Impressum Imprint}

\section{Zielsetzung der Zeitschrift}

Trauma und Berufskrankheit bietet aktuelle Fortbildung aus den Bereichen Unfall- und Wiederherstellungschirurgie, Berufserkrankungen, Arbeitsmedizin sowie Begutachtung. Die interdisziplinäre Zeitschrift richtet sich an Unfallchirurgen, Chirurgen und Orthopäden in Fachpraxen, Unfall- und Rehakliniken, Mitarbeiter der Berufsgenossenschaften und Arbeitsmediziner.

Umfassende Übersichtsarbeiten greifen ausgewählte Themen auf und bieten dem Leser eine Zusammenstellung aktueller Erkenntnisse. Im Mittelpunkt steht dabei gesichertes Wissen zu Diagnostik und Therapie mit hoher Relevanz für die tägliche Arbeit - der Leser erhält konkrete Handlungsempfehlungen. Standards in der Unfallchirurgie werden für den Leser praxisnah zusammengefasst.

Frei eingereichte Originalien ermöglichen die Präsentation wichtiger klinischer Studien und dienen dem wissenschaftlichen Austausch.

\section{Aims \& Scope}

Trauma und Berufskrankheit is a journal dealing with all aspects of trauma and reconstruction surgery, occupational diseases, occupational medicine and expertise.

The interdisciplinary journal serves both the scientific exchange and the continuing education of all specialists interested in trauma and occupational diseases.

Comprehensive reviews on a specific topical issue focus on providing evidenced based information on diagnostics and therapy.

Freely submitted original papers allow the presentation of important clinical studies and serve scientific exchange.

Review: All articles of Trauma und Berufskrankheit are reviewed.

Declaration of Helsinki: All manuscripts submitted for publication presenting results from studies on probands or patients must comply with the Declaration of Helsinki. 